\title{
Lúdica y comunidad En el desarrollo del pensamiento geométrico
}

\author{
Fabián Merardo Vega Monroy* \\ Gimeno del Carmen Ramírez Espinosa**
}

Artículo de: reflexión.

Fecha de Recepción: 30 octubre 2017.

Fecha de Aprobación: 27 febrero 2018.

\section{Resumen}

El trabajo de grado "Resignificación del Rincón de Matemáticas: una estrategia lúdica para aprender Geometría”, presentado en el programa de Maestría en Educación, Modalidad Profundización, convenio MEN-UPTC, se focalizó como una experiencia didáctica para implementar en dos cursos de dos sedes en la Institución Educativa Nueva Generación del Municipio de Sáchica. En el desarrollo del trabajo, sus autores detectaron la necesidad de trascender los espacios de las aulas de clase para involucrar a los otros estamentos de la comunidad educativa. Para dar a conocer la experiencia didáctica y lograr sensibilizar a los colegas docentes, directivos docentes, padres de familia y demás estudiantes de la institución educativa, que comprende dos sedes urbanas y cuatro sedes rurales, se programaron talleres de diseño y aplicación de materiales didácticos, un Rally y una Olimpiada de matemáticas; eventos que permitieron evidenciar cambios favorables en el ambiente de la comunidad educativa en general y una mejor disposición para el aprendizaje de la geometría en los estudiantes. En este artículo, se describe cómo se organizaron y desarrollaron
* Institución Educativa Nueva Generación, -Boyacá - (Colombia)

fabiancho42@hotmail.com

** Institución Educativa

Nueva Generación, -Boyacá

- (Colombia)

gimeno20015@gmail.com

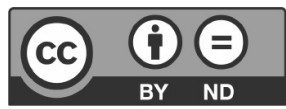


estas actividades integradoras para la comunidad educativa, identificando las dificultades que implica la participación de 630 estudiantes y las propias en cada estamento, y también cuáles fueron los logros alcanzados.
Palabras clave: matemática, geometría, enseñanza de las matemáticas, material didáctico, educación comunitaria, ambientes de la clase. 


\section{Introducción}

Al analizar los bajos resultados de las diferentes pruebas internas y externas aplicadas a los estudiantes (Instruimos, aprendamos, supérate y SABER), reflejadas en el Índice Sintético de Calidad Educativa (ISCE) 2015 y 2016, $\mathrm{y}$ al indagar por las metodologías utilizadas por los docentes en la Institución Educativa Nueva Generación del municipio de Sáchica, se puede inferir que los aprendizajes en el área de matemáticas, no son suficientemente sólidos y la mayoría de las clases se desarrollan de manera teórica. Pensando en esta situación, se propuso el proyecto "Resignificación del Rincón de Matemáticas: una estrategia lúdica para aprender Geometría”, que buscó, a través de la reorganización, diseño, elaboración y adquisición de materiales reales, virtuales e instruccionales, darle una nueva dimensión al llamado "Rincón de Matemáticas" en el programa Escuela Nueva, con la meta ambiciosa de lograr despertar el gusto y el amor por el estudio de la geometría en los estudiantes y docentes, especialmente, en el grado $5^{\circ}$ de la sede rural San Lorenzo; y el grado $6^{\circ}$, de la sede Central.

Para hacer posible el logro de estos propósitos (los materiales y los aprendizajes), fue necesario promover la vinculación de toda la comunidad educativa, mediante la participación en talleres y jornadas de sensibilización, focalizadas de manera adecuada por cada estamento. Estas actividades contaron con el respaldo y aprobación de las directivas, con el fin de institucionalizarlas e incluirlas dentro del cronograma académico de cada año, por lo que, simultáneamente, se propone interiorizar gradualmente el modelo pedagógico "Dialogante Interestructurante" adoptado por el colegio, de manera que sirva de punto de apoyo para que toda la comunidad educativa lo reconozca y lo vaya apropiando en la práctica.

En concordancia con las políticas educativas que plantean hacer de Colombia "la más educada", como docentes en el área de matemáticas en el nivel de educación básica, se propuso la creación de una cultura geométrica para generar un cambio de actitud en estudiantes, padres de familia, docentes y directivos, para mejorar el aprendizaje de la geometría y, en general, el ambiente educativo de la institución. Por esta razón, para el desarrollo del trabajo, se adoptó el modelo de investigación-acción fundamentado en el paradigma cualitativo con énfasis en la interpretación, pues tuvo como finalidad "comprender e interpretar la realidad educativa, los significados de las personas, percepciones, intenciones y acciones" (Latorre, citado en Bisquerra, 2014, p.72).

Siguiendo las fases propuestas por Elliott (1990), la investigación acción se desarrolló teniendo en cuenta los siguientes pasos: identificación e interpretación del problema, exploración y planteamiento de las acciones estratégicas a realizar, para dar solución a la problemática y la ejecución del plan de acción.
En concordancia con las políticas educativas que plantean hacer de Colombia "la más educada”, como docentes en el área de matemáticas en el nivel de educación básica 


\section{Lúdica en el Contexto Escolar}

De acuerdo con Jiménez (2005), la lúdica es importante en el proceso de aprendizaje, no es solo el juego, también involucra el reconocimiento de sí mismo y la relación con el entorno de forma armónica y agradable. La lúdica está relacionada con el pensamiento abstracto, innovador y creativo; desarrolla habilidades comunicativas y cooperativas. En cuanto el aprendizaje, la lúdica despierta la curiosidad y la imaginación, propicia una relación entre lo emocional y lo cognitivo, de esta forma, se facilita la adquisición de aprendizajes.

Durante el desarrollo de las actividades lúdicas, se observó que el estudiante disfruta participando y se motiva a dar más de sí mismo; genera mayores y mejores resultados en su aprendizaje; otra ventaja es que permite aprender del error sin traumatismos, debido a que los ambientes que se generan dan más libertad para que el estudiante actúe de manera espontánea, lo cual aumenta su confianza para participar e interactuar con sus compañeros en beneficio del trabajo colaborativo y de las relaciones interpersonales; condiciones necesarias para una buena convivencia en el ambiente escolar.

La experiencia del trabajo con la comunidad de la Institución Educativa Nueva Generación de Sáchica, en la construcción del material didáctico, ha permitido reflexionar sobre la importancia de vincular a los diferentes estamentos de la institución, donde surgen ideas innovadoras que facilitan las prácticas educativas $\mathrm{y}$, por ende, se motiva al estudiante en la construcción de sus aprendizajes. Se observa, también, que surgen intercambios de conocimientos, se genera espacios de comunicación entre los participantes, se fortalece los valores institucionales, como son: vida, respeto, tolerancia y honestidad; se da cumplimiento a los pactos y reglas establecidas en cada una de las actividades académicas. Todos estos aspectos ponen en práctica el modelo pedagógico institucional "Dialogante e Interestructurante".

Con esta propuesta, se incorpora el Rally Matemático Institucional y la Olimpiada Regional de Geometría y Estadística, como experiencias lúdicas de carácter integrador y socializador, ya que tienen como objetivo el desarrollo de valores y permiten fundamentalmente la construcción de conocimientos y aprendizajes.

\section{Pruebas internas y externas como factor de mejoramiento}

Para las autoridades educativas y para algunos docentes, los resultados de las pruebas estandarizadas reflejan la calidad de la educación. El Ministerio de Educación Nacional con el propósito de mejorar la educación, ha avanzado en cobertura escolar, pero no tanto en calidad (ISCE, 2015-2016). Para mejorar la calidad, se debe invertir en infraestructura y mantenimiento de las plantas físicas, en dotación de material didáctico, alimentación escolar, capacitación a docentes, entre 
otros, para que se pueda reflejar en los aprendizajes de los estudiantes. La prueba internacional PISA es un instrumento que mide el desempeño de los alumnos en matemática, ciencias y lectura. A nivel nacional, están las pruebas "SABER, Supérate y Aprendamos"; y en lo institucional, el colegio se encuentra inscrito a las pruebas "Instruimos", instrumento que permite una preparación previa a las pruebas nacionales.

El propósito principal de analizar los resultados de las pruebas internas y externas aplicadas en la Institución Educativa Nueva Generación, es medir la calidad de la educación, según lo plasmado en el Proyecto Educativo Institucional (2016). Los resultados obtenidos en estas evaluaciones, implican un seguimiento a los planes de estudio acorde con los Lineamientos Curriculares, los Estándares Básicos de Competencias y las metodologías utilizadas en las aulas para su enseñanza; en consecuencia, se diseña planes de mejoramiento en las diferentes áreas del conocimiento.

Al caracterizar y analizar los resultados obtenidos por los estudiantes de la institución, luego de la aplicación de algunas pruebas, se observa deficiencias en el área de matemáticas $y$, en especial, en el pensamiento geométrico (ISCE, 2015-2016). Al no superar estas deficiencias, van a presentar dificultades en el rendimiento académico en grados superiores y, posiblemente, se reflejará en la universidad para los jóvenes que aspiren continuar sus estudios. Por este motivo, se propone repensar los ambientes de aprendizaje para mejorar la calidad en la competencia geométrica en los niños; para ir superando, como valor agregado, los puntajes en los resultados de las pruebas en las que participa.

Los resultados de las diferentes pruebas internas y externas en la Institución Educativa Nueva Generación del municipio de Sáchica, tienen una utilidad pedagógica que se refleja en las estrategias de mejoramiento plasmadas en el Proyecto Educativo Institucional (PEI). Las pruebas SABER han sido, para el sistema educativo, una herramienta de reflexión, que permite comparar los resultados en los ámbitos municipal, regional y nacional e identificar las debilidades, para plantear propuestas de innovación que fortalezcan la labor docente. Los diferentes resultados no deben ser entendidos como una clasificación y estrategia de reconocimiento, sino como una oportunidad para analizar y detectar las fortalezas y las debilidades (Ministerio de Educación Nacional "MEN", 2016).

Las propuestas de mejoramiento deben ser consistentes con el contexto y contar con la participación activa de los miembros de la comunidad educativa, con el fin de lograr un crecimiento continuo en los procesos educativos. Por esta razón, este trabajo resalta la participación activa de los diferentes estamentos de la Institución Educativa Nueva Generación, con el ánimo de superar las dificultades encontradas en el área de matemáticas y, en especial, en el componente geométrico.
El propósito principal de analizar los resultados de las pruebas internas y externas aplicadas en la Institución Educativa Nueva Generación, es medir la calidad de la educación, según lo plasmado en el Proyecto Educativo Institucional (2016). 
La matemática actual debe abrir el camino para la construcción del conocimiento. En este caso, se propone que sea el componente geométrico el eje en el que deben girar los demás componentes; donde el niño pueda manipular, construir y confrontar postulados y no quedarse con una sola idea conceptual, tal vez, errónea.

EduCACIÓn y Ciencia - Núm 22. AÑo 2019 • PÁG. 489 -504

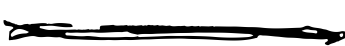

Para indagar por las causas de los bajos resultados en las pruebas, se diseñaron y aplicaron encuestas a los diferentes estamentos de la institución. Complementariamente, se construyeron cuestionarios similares a los de las pruebas SABER, Instruimos, Supérate y Aprendamos, con el objetivo de identificar, específicamente, las debilidades que presentan los estudiantes en el componente geométrico, las cuales se tomaron como soporte y referente para el diseño e implementación de estrategias para motivar y mejorar los aprendizajes y, por ende, los resultados de estas pruebas.

\section{Materiales para aprender geometría}

El docente era el único que se encargaba de la enseñanza y el alumno era un simple receptor; los problemas los proponía el maestro para que el estudiante los desarrollara; no se relacionaban con el contexto social del educando. El estudiante aprendía operaciones básicas, sin saber cuándo, dónde, por qué y para qué hacer uso de ellas.

La matemática actual debe abrir el camino para la construcción del conocimiento. En este caso, se propone que sea el componente geométrico el eje en el que deben girar los demás componentes; donde el niño pueda manipular, construir y confrontar postulados y no quedarse con una sola idea conceptual, tal vez, errónea. La matemática para el estudiante, debe convertirse en una herramienta que evolucione frente a la necesidad de encarar situaciones mediante retos y estos los deban inducir a buscar estrategias de solución, mediante el uso de sus conocimientos previos. Las situaciones problema deben estar relacionadas con el contexto cultural del niño; despertar su interés; invitarlos a reflexionar, argumentar y encontrar diferentes formas de enfrentar situaciones, gracias al uso del material didáctico concreto y software educativo relacionado con la matemática.

Los resultados de investigadores en educación que se mencionan a continuación, recomiendan procesos metodológicos que parten de lo conocido a lo desconocido y de lo concreto a lo abstracto; sin embargo, al observar los resultados de las evaluaciones internas y externas, habría que examinar la coherencia entre las prácticas educativas con estas recomendaciones, además de reconsiderar el uso de materiales como apoyo para el aprendizaje. Se comparte, a continuación, algunos aportes de los clásicos que han servido de inspiradores y puntos de apoyo para estudios recientes con plena vigencia.

Steen (1998), en "La enseñanza agradable de las matemáticas”, recuerda a Friedrich Froebel, inventor del jardín de niños ya hace casi dos siglos. Resalta que:

empezó con objetos de la parte más concreta de la matemática: esferas, cubos y cilindros. Pasó a un nivel más alto de abstracción al dar a los pequeños bandejas cubiertas con dibujos formados por mosaicos. Aumentó aún más el grado de abstracción introduciendo juegos de palitos de madera de diferentes longitudes que deberían colocarse en figuras relacionadas 
en última instancia con patrones numéricos (p.17).

Esto mismo se recomienda actualmente en los textos con énfasis didáctico y de matemática recreativa, con la finalidad de permitir el acceso de los estudiantes al fascinante mundo de las matemáticas, con la enorme posibilidad de enriquecer estos materiales con apoyo en diseños curriculares y herramientas tecnológicas.

Por otra parte, es importante retomar del mismo texto, que "los jóvenes pupilos de Froebel, pasaban gran parte de su tiempo vertiendo agua y cerniendo arena. Recipientes de formas diferentes contenían diferentes volúmenes por lo que el pequeño aprendía gradualmente relaciones comunes sin pensar siquiera en ponerlas por escrito.” (p.21), ya que deja clara la importancia de la experimentación, a manera de laboratorios sencillos, que inspirara la construcción de conceptos y relaciones.

Particularmente en matemáticas, Vasco (1994) propone un enfoque sistémico para orientar su aprendizaje y su enseñanza; propuesta que recorre tres momentos ordenados: primero, partir de los sistemas concretos o familiares para el estudiante, de manera que al interactuar con los objetos de esos sistemas concretos, pueda abstraer similitudes y diferencias que permitan la construcción de nociones, conceptos y propiedades. Segundo, manejar los sistemas conceptuales, que tienen vida en el cerebro de las personas. Tercero, asignar nombres, códigos y símbolos para representar esas construcciones abstractas: dar paso a los sistemas simbólicos. Lamentablemente, en muchos salones de clase se recorre el camino en sentido contrario, con los resultados que ya se conocen en los diferentes procesos de evaluación.

En este mismo sentido y siguiendo las ideas de Piaget (1975), el matemático canadiense Zoltan Dienes (1971) propone seis etapas para apoyar el aprendizaje en matemáticas. Comienza con la etapa de juego libre hasta llegar a la de formalización. El juego libre se apoya en la manipulación de materiales concretos, adecuados para cada estadio de desarrollo intelectual en la que se encuentre el estudiante.

La geometría, considerada como la matemática del espacio, desempeña un papel sustancial, no solo en el interior de la ciencia matemática, sino en el campo de la didáctica, al constituirse en una puerta de entrada para que los niños pequeños y los jóvenes se entusiasmen por el estudio de las matemáticas. En este campo de la geometría, también se han hecho muchos aportes para ayudar a su aprendizaje; uno de los más referenciados es el formulado por la pareja de educadores holandeses Dina y Pierre Van Hiele, a mediados del siglo pasado, más conocido como Modelo de Van Hiele (Fouz \& Donosti, 2005). Plantea unos niveles de aprendizaje y unas fases para una didáctica de la geometría que se mantienen vigentes. El primer nivel, denominado Visualización o Reconocimiento, involucra el uso y manipulación de materiales concretos o de sus representaciones, de acuerdo con la etapa de razonamiento del estudiante. Martínez y Rivaya (1989) presenta
una metodología de enseñanza de 
la geometría elemental. "Es una metodología activa y lúdica de aprendizaje por descubrimiento, basada en el juego psicomotor" (p.13). Se trata de despertar el interés espontáneo de los alumnos hacia la matemática. La lúdica, sin duda, es una estrategia atractiva en los niños, ya que les genera diversión. El docente debe aprovechar este método para orientar a sus estudiantes en la construcción de nuevos aprendizajes. Así, mejorar su capacidad de comunicación, creatividad y espontaneidad; habilidades para dar solución a diferentes situaciones cotidianas.

Según Martínez y Rivaya (1989);

El proceso de aprendizaje del alumno debe basarse en su propia actividad creadora, en sus descubrimientos personales, en sus motivaciones intrínsecas, debiendo ser la función del profesor la de un orientador, guía, animador, pero no la de fuente fundamental de información (p.17).

Cuando el estudiante profundiza de manera lógica en un determinado tema, el docente lo debe orientar a analizar situaciones más apropiadas que lo conduzcan a lograr efectivamente los aprendizajes. Los conocimientos que son construidos por los propios niños son significativos y permanentes.

Riscanevo, Suárez y Medina (2013), en la Ponencia - Simposio: "Olimpiadas de juegos inteligentes", resaltan la importancia del juego en las actividades escolares para lograr que los estudiantes se motiven en la construcción de conocimiento y el desarrollo de sus capacidades. Se presenta una dinámica en la cual se sugiere incorporar en los proyectos pedagógicos y en diferentes actividades que se programan en las instituciones, los materiales didácticos y los recursos virtuales, pues son herramientas importantes para abrir las puertas al conocimiento. En los primeros años de educación básica en el área de matemáticas, y en especial en geometría, fortalecen el desarrollo del pensamiento lógico y crítico.

Como se puede deducir de los aportes de investigadores clásicos y de los más recientes, existe consenso sobre la importancia del uso de materiales concretos, especialmente en la educación básica. También, es claro que los materiales concretos deben complementarse con otros de origen tecnológico y usar el computador y las tabletas disponibles en la institución. Las nuevas tecnologías aportan herramientas importantes y atractivas en el aprendizaje de las matemáticas, mediante software interactivo permiten relacionar de manera práctica los conceptos teóricos y motivan al estudiante a ver la matemática de forma agradable.

Con el uso de los materiales, el estudiante tiene la oportunidad de salir de la rutina, de favorecer el desarrollo de su capacidad de indagación, investigación, conjeturación y validación, de trabajar en equipo y por su propia cuenta. Con la interacción oportuna y adecuada del docente, se puede identificar y ayudar a mejorar sus actitudes y aptitudes, para que trabaje con agrado; para que construya sus aprendizajes (Jiménez, 
Limas, \& Alarcón, 2016). Por otra parte, la participación del estudiante en la elaboración del material es motivo de satisfacción cuando observa que su trabajo es incluido en el Rincón de Matemáticas o, mejor aún, cuando sus padres han sido partícipes en su construcción. Estas oportunidades de integración familiar ayudan a mejorar la comunicación y dan paso a la adquisición de nuevos aprendizajes.

En concordancia con lo expuesto, la resignificación del Rincón de Matemáticas se caracteriza por retomar las ideas planteadas por el sistema "Escuela Nueva-Escuela Activa" en lo referente a la organización de este espacio. Se trata de fortalecerlo con suficiente material para desarrollar la geometría con el objetivo, a largo plazo, de que se constituya en eje curricular para el estudio de la matemática básica. En los párrafos que siguen, se nombra algunos de los materiales adquiridos con presupuesto de la institución o que fueron construidos en actividades integradoras con la comunidad. Finalmente, no se trata de tener "un conjunto de materiales" sino, más bien, "un sistema de materiales", con descripciones, manuales de uso, categorías temáticas, guías de taller $\mathrm{y}$, a mediano plazo, guías de laboratorio.

\section{Descripción de las Actividades Realizadas}

Los estudiantes, padres de familia, docentes y directivos, participaron activamente en los talleres de elaboración del material necesario para la adecuación de los Rincones de Matemáticas. Los
Figura 1. Niños de grado $1^{\circ}$ de la sede urbana.

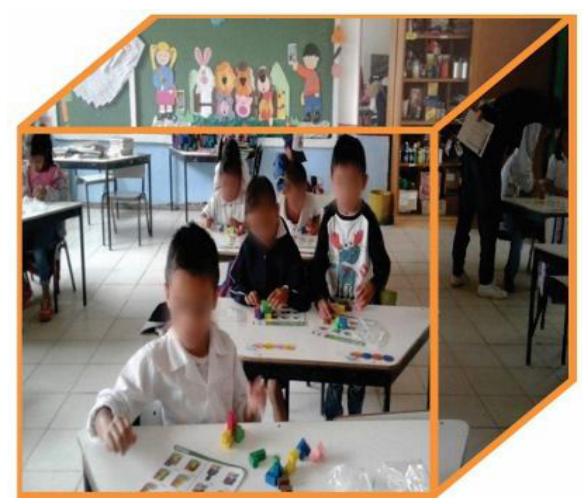

Fuente: los autores.

materiales elaborados sirvieron como herramientas fundamentales en el desarrollo de guías didácticas para lograr mejores aprendizajes en el área de matemáticas y, especialmente, para el desarrollo del pensamiento geométrico de los estudiantes.

A continuación, se describen los resultados de las estrategias utilizadas con cada uno de los estamentos de la comunidad educativa, con el ánimo de superar las dificultades encontradas en los diferentes resultados de las pruebas internas y externas; en particular, en el componente geométrico, de la Institución Educativa Nueva Generación del municipio de Sáchica, en la que se tienen como referentes los Lineamientos Curriculares, los Estándares, los Derechos Básicos de Aprendizaje y el modelo pedagógico institucional "Dialogante Interestructurante".

Taller para construir material
geométrico por parte de los
estudiantes


Desarrollado con los estudiantes de los grados $5^{\circ}$ de la sede San Lorenzo; y $6^{\circ}$, de la Sede Central, con el objetivo de elaborar material didáctico para enriquecer el Rincón de Matemáticas. En acuerdo entre docentes y estudiantes, se construyeron figuras bidimensionales y tridimensionales faltantes en los rincones, como: tangram, cubo soma, estructuras poliédricas, poliedros, polígonos regulares e irregulares, cubos multibase y decímetro cúbico. Se utilizó madera, vidrio, pitillos, palillos, papel iris, foamy, pinturas, lija, sellantes, reglas, bisturí, tijeras y pegante. Esta actividad se desarrolló durante el segundo semestre de 2016 y primer semestre de 2017. La construcción de este material permitió realimentar temas vistos en geometría: clases de polígonos, perímetros, áreas y descripción de sólidos mediante el desarrollo de guías didácticas, diseñadas por los docentes teniendo en cuenta las sugerencias de mejoramiento propuestas en el ISCE.

El material didáctico facilitó la enseñanza y el aprendizaje dentro del contexto educativo, ya que los estudiantes muestran agrado e interés al manipular material real. Estimuló los sentidos para acceder con buena actitud y de forma fácil a la adquisición de conceptos, habilidades y destrezas. Los docentes observaron el interés mostrado por los estudiantes al ayudar a construir material para el rincón de matemáticas, y el uso que se le dio para validar o refutar conceptos, nociones o propiedades. Además, facilitó a los profesores detectar actitudes y aptitudes en cada uno de sus estudiantes.
El material real construido por los estudiantes, facilita el aprendizaje a través de su manipulación y observación. Como su nombre lo indica, son los mismos objetos y no representaciones de ellos. El material con mayor validez para el estudiante, es el conjunto de trabajos, escritos, dibujos, recortes y modelos elaborados por ellos mismos o sus padres; lo anterior contribuye a darle un mejor uso y cuidado. Además, eleva su autoestima y se sienten orgullosos al ver que sus construcciones les sirven para profundizar en la materia, tanto para ellos como para sus compañeros.

Los estudiantes, luego de desarrollar las actividades, han mostrado y manifestado su interés por continuar trabajando en estos talleres lúdicos, ya que las clases han pasado de ser monótonas a ser agradables y dinámicas.

\section{Actividad de Inducción para el Rally Matemático}

Figura 3. Estudiantes de grado $4^{\circ}$ de las sedes Rurales.

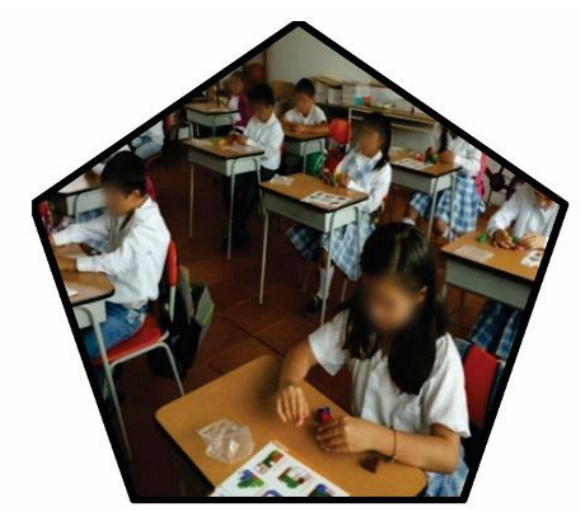

Fuente: los autores. 
Se realizaron actividades lúdicas de inducción a los niños de preescolar, primaria y secundaria, con materiales elaborados por los estudiantes de grado $4^{\circ}, 5^{\circ}$ y $6^{\circ}$, docentes y padres de familia. Esta actividad fue liderada por estudiantes del grado $11^{\circ}$ y los autores de estas líneas. Su objetivo fue prepararlos para la participación en el Rally matemático con actividades similares. Se observó gran entusiasmo al manipular diferentes materiales.

Con la colaboración de los estudiantes de grado $11^{\circ}$, fueron distribuidos por grupos y se desplazaron a las seis sedes de la Institución Educativa Nueva Generación del municipio de Sáchica. Se trabajó con guías de tangram, punto color, cubo soma, prueba escrita didáctica y otras actividades lúdicas de aprestamiento para la participación en la fase de competencia. Los resultados de la visita a las sedes rurales, fueron significativos para todos; en especial, para los estudiantes del grado $11^{\circ} \mathrm{y}$, aún más, para quienes iniciaron su primaria en estas sedes; recordaron su infancia en aquellas aulas donde crecieron. Los niños mostraron sus ganas por querer participar en esta etapa de competencia desarrollada en la sede Central. Los docentes orientadores de cada sede, se sintieron motivados por tenerse en cuenta, por primera vez, en una actividad institucional de matemáticas. Estos momentos de integración fortalecen la convivencia y ponen en práctica el modelo pedagógico institucional "Dialogante Interestructurante", las decisiones para la construcción del material y manipulación del mismo, se tomaron de forma democrática y se evidenció diálogo permanente entre estudiantes y docentes para elaborar conceptos, producir escritos, investigar y comunicar ideas matemáticas; esto ayuda a consolidar una cultura geométrica.

\section{Construcción de material geométrico por parte de docentes}

Los docentes de primaria y los de matemáticas de secundaria, participaron activamente en la construcción de figuras geométricas planas y tridimensionales con palos de madera, foamy, pitillos plásticos, porcelanicrón, hilos y pegante. Se enseñaron otros materiales para trabajar en pruebas de inducción y en el rally matemático. Esta actividad surgió por la ausencia de materiales geométricos en las diferentes sedes, razón por la cual motivó la participación de todos los docentes en esta actividad y los llevó a reflexionar sobre cada una de sus prácticas pedagógicas desarrollándolas de forma práctica y agradable; manifestaron inconformismo por la falta de tiempo

Figura 4. Docentes de la IENG.

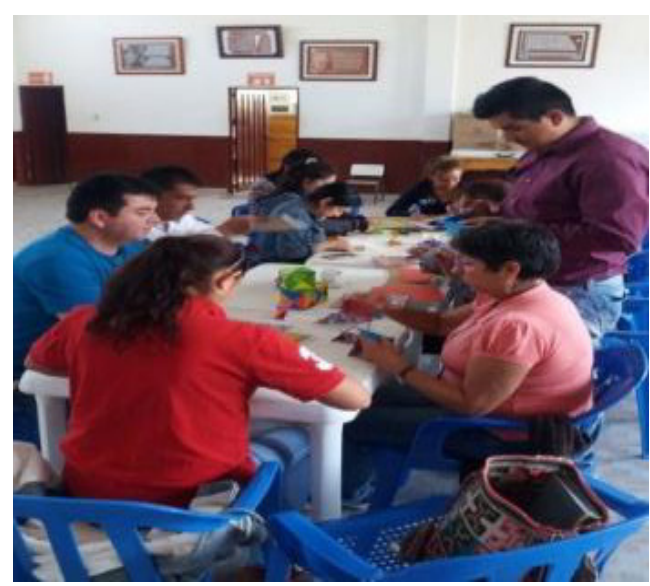

Fuente: los autores. 
y recursos para continuar elaborando materiales suficientes y necesarios para el desarrollo de las clases del área de matemáticas en sus respectivas sedes.

Construcción de material geométrico por parte de los padres de familia

Figura 5. Padres de familia de la sede San Lorenzo.

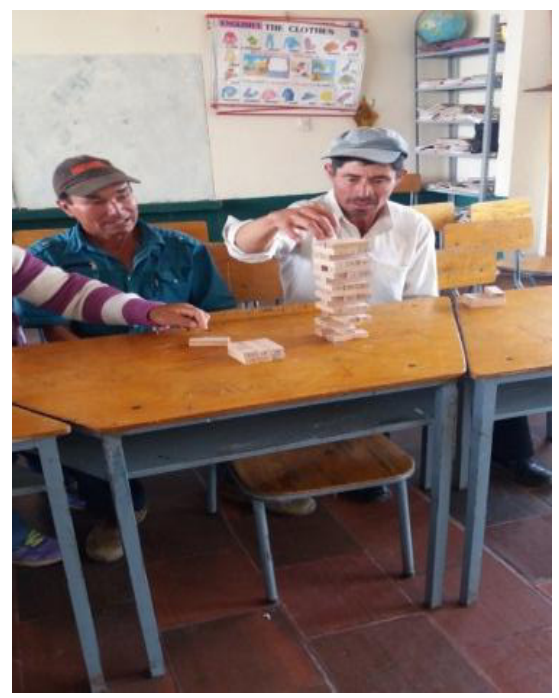

Fuente: los autores.

Con los padres de familia de la sede rural San Lorenzo, se desarrollaron dos jornadas de elaboración de diferentes materiales geométricos en madera y foamy. En esta actividad, se observó el interés de participar en el acompañamiento y ayuda a sus hijos para cumplir con sus tareas y trabajos escolares. Además, pusieron en práctica sus conocimientos y habilidades enfrentándose a diferentes retos con algunos materiales. A pesar de la falta de tiempo, por sus múltiples compromisos, trabajaron activamente y excedieron el tiempo acordado, motivados por la actividad, y solicitaron un nuevo encuentro en una jornada no laboral para ellos (día domingo).

La participación de los padres de familia, en la construcción y consecución de diferentes materiales para el rincón de matemáticas, contribuye a que ellos adquieran una mayor responsabilidad en el acompañamiento en las tareas de sus hijos. De otro lado, ellos también aprenden y ayudan en el proceso de enseñanza-aprendizaje, lo que se reflejará en gusto y amor por la materia, disciplina y mejores resultados académicos.

Si los Rincones de Matemáticas son organizados por toda la comunidad educativa, todos pueden aprender de ellos, utilizarlos, preservarlos y enriquecerlos. La organización de los rincones permite crear un ambiente amigable, donde los trabajos de los estudiantes y padres de familia son valorados, respetados y compartidos con otros, aspecto que favorece su autoestima.

Los padres de familia de los grados $6^{\circ}$ se vincularon en la elaboración de figuras geométricas, apoyados por sus hijos quienes los han motivado para continuar contribuyendo con procesos de mejoramiento académico. Este Rincón de Matemáticas cuenta con material construido por la comunidad educativa, así como con material adquirido en la empresa "Dinámico Jugando Aprendo" de la ciudad de Tunja.

La participación de los padres de familia en estas prácticas pedagógicas, 
permite desarrollar en los estudiantes, no solo habilidades comunicativas y matemáticas, sino que se construye comunidad y se fortalece el rendimiento académico, ya que los padres adquieren conocimientos en el área y, de esta forma, pueden ayudar a sus hijos en la realización de tareas. Para lograr una educación de calidad, es importante vincular a los padres de familia, como formadores y colaboradores de sus hijos en estos procesos.

\section{Participación de las directivas de la Institución}

Las directivas de la Institución mostraron su respaldo y colaboración en el desarrollo de estas actividades, y se comprometieron a institucionalizarlas incluyéndolas en el cronograma académico de cada año. La rectora facilitó algunos recursos para la compra de materiales necesarios para los talleres de manualidades.

La logística de cada uno de los momentos en el desarrollo del trabajo, contó con el direccionamiento de la Rectoría, Coordinación Académica y Disciplinaria quienes, a su vez, valoraron y resaltaron los resultados obtenidos al finalizar cada jornada. Por otra parte, se resalta el compromiso en cuanto la autorización de los espacios y tiempos que han sido necesarios para lo pertinente.

\section{Actividad decompetencia: Rally Matemático}

Figura 6. Estudiantes grado $6^{\circ}$ en prueba de competencia.

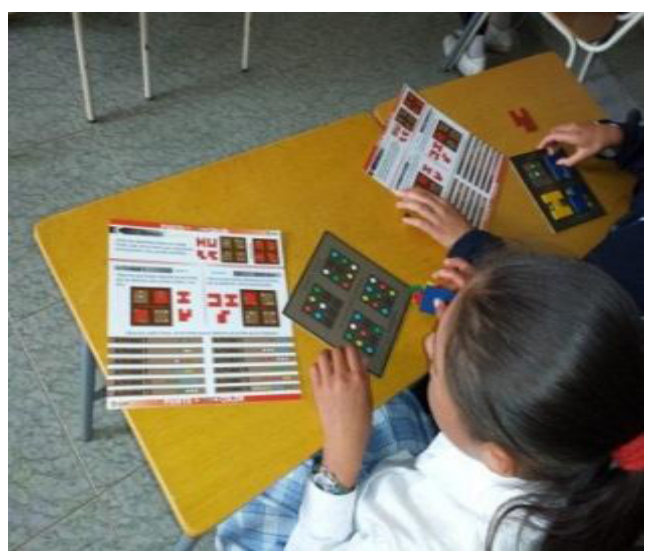

Fuente: los autores.

El pasado 31 de agosto de 2016, se llevó a cabo el "Rally matemático" organizado por los docentes del área, quienes cursan la Maestría en Educación, modalidad Profundización, en convenio MEN-UPTC; encargados de la logística de la actividad, con la colaboración de los estudiantes del grado $11^{\circ}$ de la Institución Educativa Nueva Generación de Sáchica y el grupo "Dinámico Jugando Aprendo", de la ciudad de Tunja, quienes facilitaron algunos materiales didácticos que se complementaron con el material elaborado por los estudiantes, padres de familia y docentes de la institución para esta importante actividad didáctica recreativa.

Esta jornada se llevó a cabo en la sedes de primaria y bachillerato, con la participación de las escuelas rurales: San Lorenzo, Quebrada Arriba, Arrayán y Espinal, la sede central y colegios privados del municipio: Santo Domingo y Mi Pequeño Mundo (hoy llamado María Montessori); se contó con el apoyo incondicional de todos los docentes y directivos de la institución, quienes se vincularon con la actividad.
La participación de los padres de familia en estas prácticas pedagógicas, permite desarrollar en los estudiantes, no solo habilidades comunicativas y matemáticas, sino que se construye comunidad y se fortalece el rendimiento académico, ya que los padres adquieren conocimientos en el área y, de esta forma, pueden ayudar a sus hijos en la realización de tareas. 
El resultado de estas competencias cumplió las expectativas de los organizadores. La actividad estuvo distribuida en 4 sesiones, con una duración de seis horas en total. Todos los estudiantes participaron en una prueba escrita, con una duración de una hora; luego, actividades lúdicas denominadas: punto color y construcción de figuras con piezas de cubo soma y tangram, con una duración de tres horas, acorde a una guía con instrucciones. Finalmente, el Rally matemático, que contaba con veinte pistas para cada equipo de 3 estudiantes, las cuales se encontraban distribuidas en diferentes lugares de la planta física, duró dos horas.

Se contó con la participación de 630 estudiantes de las diferentes sedes, quienes se destacaron por su dinamismo en el desarrollo de cada una de las pruebas. Los estudiantes de cada curso que obtuvieron las puntuaciones más altas, recibieron una mención de honor, entregada en la ceremonia institucional de clausura.

Con esta actividad, se pretende invitar, motivar y vincular a todos los docentes de las sedes, a resignificar los rincones de matemáticas que contengan materiales didácticos para el uso de actividades de geometría y que sirvan como herramientas en la construcción de aprendizajes. Para contribuir con el mejoramiento de los resultados de las diferentes pruebas internas y externas.

Teniendo en cuenta los resultados exitosos, manifestados por los miembros de la comunidad educativa, se ha establecido como una actividad institucional de todos los años. Para este año, se llevará a cabo el 30 de agosto. Se tendrán en cuenta las falencias encontradas en el Rally anterior para corregirlas. Adicionalmente, se está organizando una Olimpiada Regional de Geometría y Estadística, para el 27 de septiembre del presente año, en la que se invitará a las instituciones educativas de los municipios de la provincia de Ricaurte Alto. Proyecto impulsado por los docentes autores de este artículo.

Dentro de la organización, para este año se tiene prevista la participación en el Rally de matemáticas, con una muestra de padres de familia de la Institución, para quienes deseen poner a prueba sus capacidades y destrezas en el desarrollo de actividades didácticas, las cuales no necesitan de la aplicación de conceptos técnicos. Se busca integrar a todos los estamentos de la institución, y mejorar, así, las relaciones interpersonales y comunicativas.

El objetivo de la Olimpiada Regional de Geometría y Estadística, es hacer de esta institución el centro de estudio y presentación del proyecto: "Resignificación del Rincón de Matemáticas: una estrategia Lúdica para aprender Geometría" y motivar a docentes y estudiantes para que profundicen en el estudio de estas dos disciplinas de la matemática, en donde la mayoría de las instituciones educativas han obtenido bajos resultados en las diferentes pruebas. Para esta actividad, se tiene previsto invitar a catorce municipios de la región: Samacá, Cucaita, Sora, Iguaque, Villa de Leyva, Moniquirá, Gachantivá, Arcabuco, Santa Sofía, Sutamarchán, 
Tinjacá, Ráquira, San Miguel de Sema, y Chiquinquirá. Para este encuentro, se cuenta con el apoyo del grupo "Dinámico Jugando Aprendo".

\section{Conclusiones}

En general, todos los estamentos de la Institución Educativa Nueva Generación, durante el desarrollo de cada una de las actividades, mostraron una participación activa, agradable, disciplinada e integradora; siguieron las reglas establecidas.

En el desarrollo de cada una de las actividades, se evidenció un cambio de actitud en las relaciones interpersonales y facilitó el trabajo en equipo. Los estudiantes de la Institución han logrado familiarizarse con la manipulación de material concreto, la elaboración de conceptos y significados de temas geométricos. Han aumentado su interés por las clases de matemáticas.

Los docentes comprometidos con esta propuesta, hemos generado e implementado un cambio en la educación de nuestros estudiantes, especialmente, en las clases de matemáticas, abandonando las clases rutinarias y teóricas, para pasar a unas clases dinámicas y participativas, en donde el estudiante construye su propio conocimiento gracias al uso y manipulación de diferentes materiales geométricos, tanto concretos como de software.

La organización de los rincones de matemáticas en la Institución Educativa Nueva Generación, sede San Lorenzo y sede Central, con la Resignificación que se adelanta, ha permitido motivar a toda la comunidad para que vean la matemática como una materia práctica y agradable, aspectos que se han evidenciado en el desarrollo de cada uno de los talleres.

Continuamente, han surgido ideas innovadoras que enriquecen y ayudan a cumplir los objetivos planteados. Este trabajo es recompensado al evidenciar que estudiantes y demás miembros de la comunidad han reflejado y manifestado motivación, agrado, gusto y amor en el desarrollo de las diferentes actividades.
En el desarrollo de cada una de las actividades, se evidenció un cambio de actitud en las relaciones interpersonales y facilitó el trabajo en equipo. 


\section{Referencias}

Bisquerra, A. (2014). Metodología de la Investigación Educativa (4ta ed.). Madrid: Editorial la muralla.

Colombia Aprende. (2016). ISCE (Índice Sintético de Calidad Educativa). Recuperado de http://aprende.colombiaaprende.edu.co/es/siemprediae/86402.

Dienes, Z. (1971. Las Seis Etapas del Aprendizaje de las Matemáticas. Barcelona: Editorial Teide.

Elliot, J. (1990). La Investigación-Acción en Educación. Londres: Ediciones Morata.

Fouz, F., \& Donosti, B. (2005). Modelo de Van Hiele para la Didáctica de la Geometría. Un paseo por la geometría 2004-2005. España: UPV-EHU. Leioa.

Jiménez, A., Limas, L., \& Alarcón, J. (2016). Prácticas pedagógicas matemáticas de profesores de una institución educativa de enseñanza básica y media. Praxis $\mathbb{\sigma}$ Saber, 7(13), 127-152. https://doi.org/10.19053/22160159.4169

Jiménez, C. (2005). Pedagogía lúdica: El taller Cotidiano y sus Aplicaciones. Armenia: Kinesis.

Martínez, A., \& Rivaya, F. (1989). Una Metodología Activa y Lúdica para la Enseñanza de la Geometría 16. Vallehermoso, Madrid: Editorial Síntesis, S. A.

Ministerio de Educación Nacional - MEN. (2016). Informe Nacional sobre el Desarrollo de la Educación en Colombia. Recuperado de https://www. mineducacion.gov.co /1621/article-107411.html

Piaget, J. (1975). Seis Estudios de Psicología. Barcelona, España: Barral editores.

Proyecto Educativo Institucional - PEI. (2016). Documento de la Institución Educativa Nueva Generación. Sáchica Boyacá. Colombia.

Riscanevo, L., Suárez, P., \& Medina, J. (2013). Ponencia - Simposio, Olimpiadas de juegos inteligentes. Recuperado de http://es.calameo.com/ $\mathrm{read} / 000549213 \mathrm{~b} 323970 \mathrm{adc} 8 \mathrm{e}$

Steen, L. (Ed.). (1998). La Enseñanza Agradable de las Matemáticas. Balderas, México, D.F.: Editorial Limusa, S.A.

Vasco, C. (1994). Un nuevo enfoque para la didáctica de las matemáticas. Serie Pedagogía y Currículo, Vol. I. Bogotá: Ministerio de Educación Nacional. 